\title{
913 A MURINE GASTRIC CANCER YTN16 MODEL FOR THE RATIONAL DESIGN OF COMBINATION IMMUNOTHERAPY
}

Koji Nagaoka*, Changbo Sun, Yukari Kobayashi, Kazuhiro Kakimi. The University of Tokyo Hospital, Tokyo, Japan

Background Although Immune checkpoint blockade (ICB) has changed the standard of care of cancer, ICB monotherapy is largely ineffective in patients with solid cancer. Therefore, the rational combination of ICB with other treatment modalities is warranted. We have recently established chemically induced gastric cancer cell line YTN16, transplantable in immune-competent mice, ${ }^{1}$ and showed that inhibition of FGFR4 signaling suppressed the YTN16 tumor growth. We also demonstrated the depletion of IL-17-producing T cells in YTN16 tumors enhanced the anti-tumor effects of anti-PD-1 treatment. ${ }^{2}$ In this study, we identified the neoantigens of YTN16 to monitor the dynamics of tumor-specific $\mathrm{CD} 8<\sup >+</$ sup $>$ T cells in response to combination immunotherapy.

Methods Whole-exome and transcriptome sequencing analyses were performed to identify missense mutations. As a result, candidate neoepitopes were predicted as follows; FPKM $\square 25$, variant allele frequency in RNAseq $\square 0.04$, IC50 of NetMHCpan $\square 250 \mathrm{nM}$, EL rank of NetMHCpan $\square 0.5$ and presentation percentile of MHCflurry $\square 0.5$.

Results Exome sequencing identified 3,347 missense mutations in the YTN16 tumor. We synthesized 11 candidate neoepitope peptides and screened their reactivity to YTN16-reactive CD8 $<$ sup $>+</$ sup $>$ T cell lines established from YTN16rejected mice by ICB. Out of 11 peptides, five peptides (3 neoantigens, m(mutated)Cdt1, mScarb2 and mZfp106) induced IFN $\gamma$ production in YTN16-reactive $\mathrm{CD} 8<$ sup $>+<$ /sup $>$ $\mathrm{T}$ cells. MHC class I dimer assay identified these three neoantigen-specific $\mathrm{T}$ cells in YTN16 tumors. Anti-CTLA-4, but not anti-PD-1 increased neoantigen-specific $\mathrm{T}$ cells and completely eradicated tumors. Adoptive transfer of neoantigen-reactive CD $8<\sup >+</$ sup $>$ T cell lines and therapeutic vaccines of DCs pulsed with neoantigen short or long peptides inhibited YTN16 growth. Neoantigen-specific TCRs were cloned from neoantigen-reactive $\mathrm{CD} 8<\sup >+</$ sup $>\mathrm{T}$ cell lines and retrovirally transduced to activated $\mathrm{CD} 8<\sup >+</$ sup $>\mathrm{T}$ cells. TCR-transduced T cells killed YTN16 in vitro and adoptive transfer of TCR-transduced T cells showed anti-tumor effects. These results indicated that these three neoantigens were de fact neoantigens.

Conclusions We identified three neoantigens that induced CD8 $<\sup >+</$ sup $>\mathrm{T}$ cell response with anti-tumor effects in YTN16. Thus, YTN16 is a well-characterized murine gastric cancer model for rational design and optimization of combination immunotherapy.

\section{REFERENCES}

1. Yamamoto $M$, Nomura $S$, Hosoi $A$, Nagaoka $K$, lino $T$, Yasuda $T$, Saito $T$, Matsushita H, Uchida E, Seto Y, Goldenring JR, Kakimi K, Tatematsu M, Tsukamoto T. Established gastric cancer cell lines transplantable into $\mathrm{C} 57 \mathrm{BL} / 6$ mice show fibroblast growth factor receptor 4 promotion of tumor growth. Cancer Sci 2018;109(5):1480-1492.

2. Nagaoka K, Shirai M, Taniguchi K, Hosoi A, Sun C, Kobayashi Y, Maejima K, Fujita M, Nakagawa H, Nomura S, Kakimi K. Deep immunophenotyping at the single-cell level identifies a combination of anti-IL-17 and checkpoint blockade as an effective treatment in a preclinical model of data-guided personalized immunotherapy. J Immunother Cancer 2020;8(2):e001185.

http://dx.doi.org/10.1136/jitc-2021-SITC2021.913 